OPEN ACCESS

Edited by:

Maria Carlota Vaz Patto, Universidade Nova de Lisboa,

Portugal

Reviewed by:

Chad Niederhuth,

University of Georgia, USA

Coralie Lashbrook,

Des Moines Area Community College,

USA

John Walker,

University of Missouri, USA

*Correspondence:

Reidunn B. Aalen

reidunn.aalen@ibv.uio.no

${ }^{\dagger}$ Joint first authors.

Specialty section:

This article was submitted to Crop Science and Horticulture,

a section of the journal

Frontiers in Plant Science

Received: 14 July 2015 Accepted: 15 October 2015 Published: 30 October 2015

Citation:

Sto IM, Orr RJS, Fooyontphanich K,

Jin X, Knutsen JMB, Fischer U, Tranbarger TJ, Nordal I and Aalen RB (2015) Conservation of the abscission

signaling peptide IDA during

Angiosperm evolution: withstanding genome duplications and gain and

loss of the receptors HAE/HSL2.

Front. Plant Sci. 6:931.

doi: $10.3389 /$ fpls.2015.00931

\section{Conservation of the abscission signaling peptide IDA during Angiosperm evolution: withstanding genome duplications and gain and loss of the receptors HAE/HSL2}

\author{
Ida M. Sto ${ }^{1+}$, Russell J. S. Orr ${ }^{1 \dagger}$, Kim Fooyontphanich ${ }^{2}$, Xu Jin ${ }^{3}$, Jonfinn M. B. Knutsen ${ }^{1}$, \\ Urs Fischer ${ }^{3}$, Timothy J. Tranbarger ${ }^{2}$, Inger Nordal ${ }^{1}$ and Reidunn B. Aalen ${ }^{1 *}$ \\ 'Section for Genetics and Evolutionary Biology, Department of Biosciences, University of Oslo, Oslo, Norway, ${ }^{2}$ UMR \\ Diversité et Adaptation et Développement des Plantes, Institut de Recherche pour le Développement, Montpellier, France, \\ ${ }^{3}$ Department of Forest Genetics and Plant Physiology, Umeå Plant Science Centre, Swedish University of Agricultural \\ Sciences, Umeå, Sweden
}

The peptide INFLORESCENCE DEFICIENT IN ABSCISSION (IDA), which signals through the leucine-rich repeat receptor-like kinases HAESA (HAE) and HAESA-LIKE2 (HSL2), controls different cell separation events in Arabidopsis thaliana. We hypothesize the involvement of this signaling module in abscission processes in other plant species even though they may shed other organs than $A$. thaliana. As the first step toward testing this hypothesis from an evolutionarily perspective we have identified genes encoding putative orthologs of IDA and its receptors by BLAST searches of publically available protein, nucleotide and genome databases for angiosperms. Genes encoding IDA or IDA-LIKE (IDL) peptides and HSL proteins were found in all investigated species, which were selected as to represent each angiosperm order with available genomic sequences. The 12 amino acids representing the bioactive peptide in $A$. thaliana have virtually been unchanged throughout the evolution of the angiosperms; however, the number of IDL and HSL genes varies between different orders and species. The phylogenetic analyses suggest that IDA, HSL2, and the related HSL1 gene, were present in the species that gave rise to the angiosperms. HAE has arisen from HSL1 after a genome duplication that took place after the monocot-eudicots split. HSL1 has also independently been duplicated in the monocots, while HSL2 has been lost in gingers (Zingiberales) and grasses (Poales). IDA has been duplicated in eudicots to give rise to functionally divergent IDL peptides. We postulate that the high number of IDL homologs present in the core eudicots is a result of multiple whole genome duplications (WGD). We substantiate the involvement of IDA and HAE/HSL2 homologs in abscission by providing gene expression data of different organ separation events from various species.

Keywords: LRR-RLK, phylogeny, genome duplication, peptide signaling, leaf abscission, fruit abscission, Populus, oil palm 


\section{INTRODUCTION}

Plant architecture is dependent on the balance between cell division and cell elongation, proliferation and differentiation, as well as formation and abscission of organs. Plants are sessile organisms that over evolutionary time have adapted to their environment. They have developed reproductive strategies involving mechanisms that in various ways facilitate fruit and seed dispersal. The shedding of organs that have served their purpose is furthermore part of normal development, but detachment can also be a response to injury or environmental changes. The model plant $A$. thaliana shed individual floral organs (petals, sepals, and stamen) shortly after pollination (Bleecker and Patterson, 1997), and displays opening of the valves of the mature siliques (so called dehiscence), and thereafter seed dropping. Other species may shed leaves (e.g., deciduous trees like aspen, Populus tremula) and whole flowers (e.g., Citrus), or fruits (e.g., tomato, palms).

Despite variation in the sites of abscission in different species of flowering plants, various abscission events are similar at the cellular level in that they take place in specialized abscission zones $(A Z s)$. AZs are either generated already during the development of the organ, or can be induced in response to hormonal or environmental cues (Patterson, 2001; Roberts et al., 2002; Lewis et al., 2006; Aalen, 2011). Organ detachment is a cell separation process that involves dissolution of the middle lamella between adjacent AZ cell files presumably through the action of a number of different cell wall remodeling and degrading proteins, e.g., polygalacturonases, expansins, XTHs, and endoglucanases (Cho and Cosgrove, 2000; González-Carranza et al., 2007; Lashbrook and Cai, 2008; Meir et al., 2010; Liu et al., 2013; Niederhuth et al., 2013; Tsuchiya et al., 2015). One can assume that there is a need for tight regulation and coordination of expression of genes involved in cell separation processes, since cell wall weakening and breakdown could render the plant more susceptible to pathogen attack, and premature organ loss would reduce reproductive success.

In A. thaliana a peptide ligand-receptor system responsible for such tight regulation of floral organ abscission has been identified (Butenko et al., 2003; Stenvik et al., 2008). This signaling module consists of the secreted peptide IDA encoded by the gene INFLORESCENCE DEFICIENT IN ABSCISSION (At1g68765), and the two leucine-rich repeat (LRR) receptorlike kinases (RLK) HAESA (HAE) and HAESA-LIKE2 (HSL2) (Cho et al., 2008; Stenvik et al., 2008). In both ida and hae hsl2 mutants floral organs are retained indefinitely. IDA belongs to a small gene family in $A$. thaliana with five additional IDALIKE (AtIDL) members that to a varying degree can substitute for IDA function (Butenko et al., 2003; Stenvik et al., 2008). IDA and the IDL proteins share a short C-terminal sequence encompassing the bioactive peptide (Stenvik et al., 2008), which for IDA has been delineated to a 12 amino acids (aa) long prolinerich peptide (hereafter called mature IDA, mIDA) using genetic and biochemical tools (Stenvik et al., 2008; Butenko et al., 2014). Overexpression either of IDA or AtIDL genes, results in early abscission and enlarged abscission zones (AZ) at the base of the floral organs, but when expressed using IDA's own promoter, only IDA and AtIDL1 can fully complement the ida mutant phenotype. Together this may suggest that AtIDL2-5 peptides have lower affinity to HAE and HSL2, and possibly signal through (a) different, but related receptor(s) (Stenvik et al., 2008). There are more than 600 genes in A. thaliana encoding receptor-like kinases, and of these more than 200 encode extracellular domains with LRRs of varying length assumed to bind small peptides (Shiu and Bleecker, 2001). Only about a dozen of these have been matched with a ligand (Butenko et al., 2009). The majority of these, including HAE and HSL2, belong to the RLK subclass XI, which has more than 20 LRRs.

Interestingly, ectopic expression of IDA in A. thaliana leads to abscission of fruit, flowers, branches, and cauline leaves at vestigial $\mathrm{AZ}$ at the base of these organs, which are sites of cell separation in a number of other species, but normally not in $A$. thaliana (Stenvik et al., 2006). Additionally, the IDA-HAE/HSL2 signaling module is involved in a quite different cell separation process, namely lateral root emergence (Kumpf et al., 2013). IDA and the receptors are expressed in the endodermal, cortical, and epidermal cells overlaying lateral root primordia, induce expression of cell wall remodeling genes and facilitate emergence without cellular disruption (Aalen et al., 2013; Kumpf et al., 2013).

The objective of the presented work has been to investigate to what extent orthologs of IDA, HAE, and HSL2 are present in other angiosperms irrespective of which organs they shed, and whether gene expression data could substantiate a role of these orthologs in cell separation events.

\section{MATERIALS AND METHODS}

\section{Phylogenetic Analyses}

Sequences used in this study were obtained from various databases [NCBI-protein, NCBI-Assembly, Phytozome, Ancestral Angiosperm Genome Project (AAGP), Comparative Genomics (CoGe)] using either tBLASTn or BLASTp (as of 3.2015) with an A. thaliana IDL or HSL query. Outgroup taxa for both monocot and eudicot receptors were closely related $A$. thaliana representations within the subclass XI LRR-RLKs. IDA was inferred without an outgroup. An aa alignment ( $\sim 59$ aa) of angiosperm IDA without the N-terminal secretion signal and LRR-RLK group (full length 1926 aa) was constructed using MAFFTv6 E- INS-I model under default settings (Katoh and Toh, 2008). The resulting alignments were checked with Gblocks v0.91b (Castresana, 2000), under the least stringent parameters (small final block, gap positions in final block and less strict flanking), to exclude poorly aligned positions and divergent regions from subsequent phylogenetic inferences. Multiple homologous copies of HSL1/HSL2/HAESA (At1g28440/At5g65710/At4g28490) for each species were removed to reduce phylogenetic noise; in all cases the copy with most sequence data and thereafter shortest branch length was retained for further analysis. ProtTest v2.4 (Abascal et al., 2005) determined LG as the optimal evolutionary model. Maximum Likelihood (ML) analyses were performed with RAxML-VI-HPCv8.0.26, using the PROTCATLG model with 25 
rate categories (Stamatakis, 2006). The most likely topology was established from 100 separate searches and bootstrap analyses were performed with 500 pseudoreplicates.

All model estimation and phylogenetic analyses were done using either Lifeportal (https://lifeportal.uio.no) or the abel server at the University of Oslo. All alignments constructed as part of this study are available as Supplementary Data sets, and will in addition be made freely available as Supplementary resources through the authors' Research Gate pages (https:// www.researchgate.net/profile/Reidunn_Aalen) and (https:// www.researchgate.net/profile/Russell_Orr2).

\section{Plant Material}

A. thaliana has been transformed with HAE and HSL2 promoter::GUS constructs in the vector pMDC162 made using Gateway Cloning Technology (Curtis and Grossniklaus, 2003) with the same promoter region upstream of the coding sequence (1601 bp and $2300 \mathrm{bp}$, respectively) as previously reported for HAE and HSL2 constructs with YFP reporter (Kumpf et al., 2013).

Fully expanded leaf blades of hybrid aspen, Populus tremula L. X P. tremuloides Michx.; clone T89, were shaded in aluminum foil to induce abscission and total RNA was extracted from $3 \mathrm{~mm}$ thick leaf axils 6 days after shading started using RNeasy Plant Mini Kit (Qiagen). Two micrograms of total RNA was used as a template for reverse transcription with the QuantiTect Reverse Transcription Kit (Qiagen). Quantitative real-time PCR (qPCR) was performed using SYBR Green I Master in combination with a LC4800 (Roche Diagnostics) qPCR machine. Primers are specified in Supplementary Table S1. Expression was normalized to PtACTIN1 expression.

mRNA was isolated from AZs of unripe and ripe of oil palm (Elaeis guineensis) fruit, and AZs of fruit treated with ethylene as described previously (Roongsattham et al., 2012). Primers were designed to fit the various EgIDA genes, EgHSL2, and EgHSL1 (Supplementary Table S1). qPCR was performed as previously described (Roongsattham et al., 2012). All expression was normalized to the EgEf 1 (accession number: AY550990) expression. No change of EgEf $\alpha 1$ transcript accumulation was found in the fruit tissues treated or not treated with ethylene. Controls were conducted to validate the absence of DNA in each sample.

\section{RESULTS}

\section{HSL2 has been Lost in the Gingers and the Grasses, and HAE is Present only in Eudicots}

The A. thaliana HAE and HSL2 receptors are closely related (60\% similar and $45 \%$ identical aa) but HAE is even more closely related to HSL1 (73\% similarity, 58\% identical aa), an RLK so far with unknown function. BLASTp and tBLASTn were used to identify putative HSL1 angiosperm orthologs in addition to orthologs of HAE and HSL2. Other related receptors from the LRR-RLK subclass XI were used as outliers. Protein sequences from 60 species belonging to 24 orders covering basal angiosperms, monocots, basal eudicots, and core eudicot clades were used to determine the phylogenetic relationship.

The LRR-RLK ingroup is monophyletic, separated from the outgroup with high support (98\% Bootstrap, BP) (Figure 1, Supplementary Figure S1). HSL2 and HSL1/HAE represent two fully supported distinct clades (100\% BP). In the HSL2 clade, the basal angiosperm copy is first to diverge within a fully supported grouping, leaving a highly supported (92\% BP) monocot/eudicot clade. Subsequently, a moderately supported (71\% BP) monocot clade diverges, leaving a poorly supported (54\% BP) eudicot monophyly. HSL2 is however missing in the taxa from the orders Poales and Zingiberales (Supplementary Figure S1).

The HSL1/HAE clade is fully supported with the basal angiosperm copy being first to diverge leaving a moderately supported ( $84 \%$ BP) monocot/eudicot clade (Figure 1). The monocot HSL1 copy diverges next forming a highly supported clade (96\% BP). Two groupings are found within the monocot clade; the first-constituting all monocot orders (HSL1A) - is unsupported (40\% BP), but excluded from the second, longerbranching HSL1B. This duplicate HSL1 clade for the orders Arecales (palms), Poales, and Zingiberales, has moderate support (72\% BP) (Figure 1, Supplementary Figure S1). The eudicot HSL1/HAE grouping is moderately supported (70\% BP). The HAE clade, representing Vitales in addition to all Rosids and Asterids, is moderately supported ( $80 \% \mathrm{BP}$ ) and excludes the basal eudicot orders Ranunculales, Proteales, and Caryophyllales which have a closer affinity to the moderately supported $(82 \%$ BP) eudicot HSL1 grouping. The fully supported Caryophyllales clade forms a moderately supported (80\% BP) monophyly with the eudicot HSL1 grouping.

Our analyses revealed that all angiosperms have HSL1 genes, in in basal angiosperms and some monocots, and almost all eudicots in combination with a HSL2 gene. Only the core eudicots have both a HAE and a HSL2 gene. In A. thaliana $H A E$ and HSL2 are redundant in function with respect to floral organ abscission, as both genes must be mutated to give the abscission-deficient phenotype (Cho et al., 2008; Stenvik et al., 2008).

\section{HAE and HSL1 Differ from HSL2 Especially in their Kinase Domains}

LRR-RLK proteins consist of three domains with different functions: the intracellular kinase domain that confers the signaling output upon ligand binding, the transmembrane domain that anchors the receptors in the plasma membrane, and the extracellular LRR to which the ligand will bind (called the ectodomain). As these three domains have different functions, the selective pressure for rejecting or preserving new mutations might differ.

The intracellular kinase domain has typically two regions: the N-terminal lobe with several $\beta$ strands and a conserved ATP binding site, and the larger $\mathrm{C}$-terminal part with a number of $\alpha$-helices, and an activation loop with serine/threonine [Ser(S)/Thr(T)] target residues for phosphorylation. In the BRASSINOSTEROID INSENSITIVE1 (BRI1) LRR-RLK several such residues are phosphorylated (Wang et al., 2005). These residues are well conserved in the $A$. thaliana 


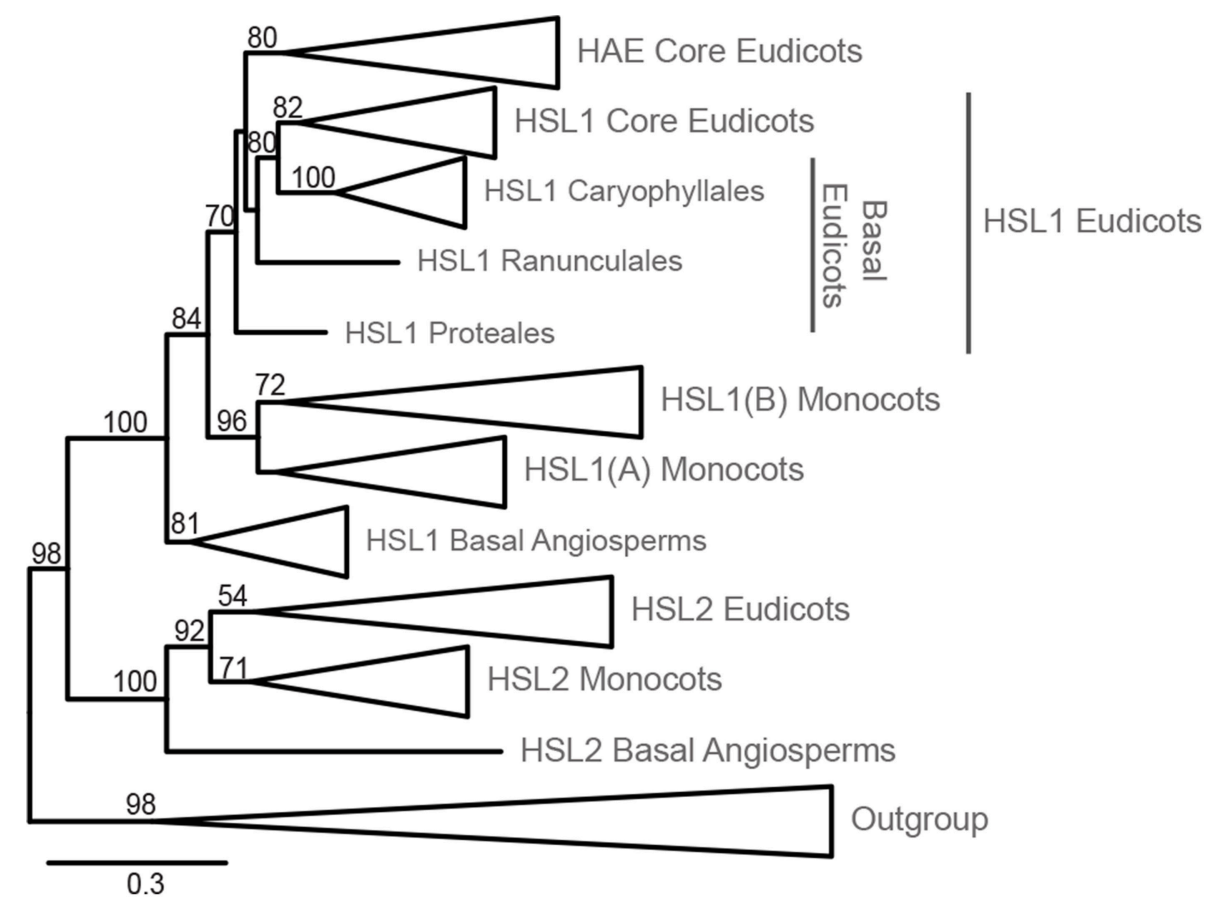

FIGURE 1 | Phylogeny of HSL LRR-RLK evolution within Angiosperms. Phylogeny inferred from ML with 145 ingroup taxa and 784 amino acid characters. The phylogeny has been collapsed at different taxonomic levels and shows only bootstrap values $>50$. The expanded tree is presented in Supplementary Figure S1, which includes an overview of species comprising each order. The alignments used for the construction of this phylogeny are available as Supplementary Data Sheet 1 and 2.

HAE, HSL1 and HSL2 receptors and their orthologs in other species (Supplementary Figure S2A). This includes the residue corresponding to $\mathrm{S} 861$ of $\mathrm{HAE}$, which is subject to autophosphorylation (Taylor et al., 2013), and several residues predicted to be targets of Ser/Thr kinases (e.g., by using PhosPhAt http://phosphat.uni-hohenheim.de/) (Durek et al., 2010). However, in loops between helices positions of putative phosphorylation sites differ in HSL2 receptors compared to HAE/HSL1. Furthermore, HSL2 receptors lack a C-terminal extension with putative phosphorylation sites (Supplementary Figure S2A). The predicted secondary structures of the transmembrane domains of HAE, HSL1, and HSL2 are similar, with a membrane-spanning, hydrophobic $\alpha$-helix flanked by conserved aa (Supplementary Figure S2B). HSL2 sequences differ from HAE and HSL1 sequences by a shorter $\alpha$-helix. HAE sequences can be distinguished from HSL1 by a two aa deletion near the end of the transmembrane domain (Supplementary Figure S2B).

So far, the exact location of the $A$. thaliana mIDA peptide when binding HAE or HSL2 is not known, however, in other cases small peptides have been shown to bind consecutive repeats on the inside pocket of the LRR (Sun et al., 2013). Each repeat has typically 24 residues with six evenly distributed conserved leucine residues (Leu, L), which together with asparagine (Asn, N) and glycine (Gly, G) in conserved positions build the structural framework of the ectodomain (Figure 2). These residues are almost invariable and have therefore limited informative value in a phylogenetic perspective. In contrast, conserved non-structural residues are interesting since selection pressure conserving residues involved in ligand binding is likely. To identify such residues we employed the Repeat Conservation Mapping (RCM) (http://144.92.198.58/main/main.php), which relies on a set of algorithms to identify predicted functional sites of LRR domains. This is accomplished by identifying the extent of conservation of different aa patches on the predicted surface of LRR and generating a colored heat map, displaying how conserved an aa is in a given position in a set of orthologs (Helft et al., 2011). For all the HSL receptors a region with highly conserved aa was stretching from the seventh LRR with the common signature sequence QIEL[Y,F], to the fourteenth LRR (Figure 2). The signatures were more similar between the HAE and HSL1, than HSL2.

\section{The Bioactive IDA Peptide is Conserved in all Flowering Plants}

IDA and IDL are secreted peptides generated from prepropeptides (Figure 3A), where the hydrophobic Nterminus is a signal directing the protein to the secretory pathway. The variable middle part and the C-terminus are assumed to be cleaved off in the apoplastic space to release a mature 12 aa peptide named PIP after the three first residues, PIPPSAPSKRHN (Butenko et al., 2003). Synthetic mIDA peptide with hydroxylation on the central proline (Pro, P) can bind and activate HSL2 efficiently, and HAE at a higher concentration (Butenko et al., 2014). The Pro residues in positions 2, 3, and 7 , serine (Ser, S) in positions 5 and 10 , and histidine 

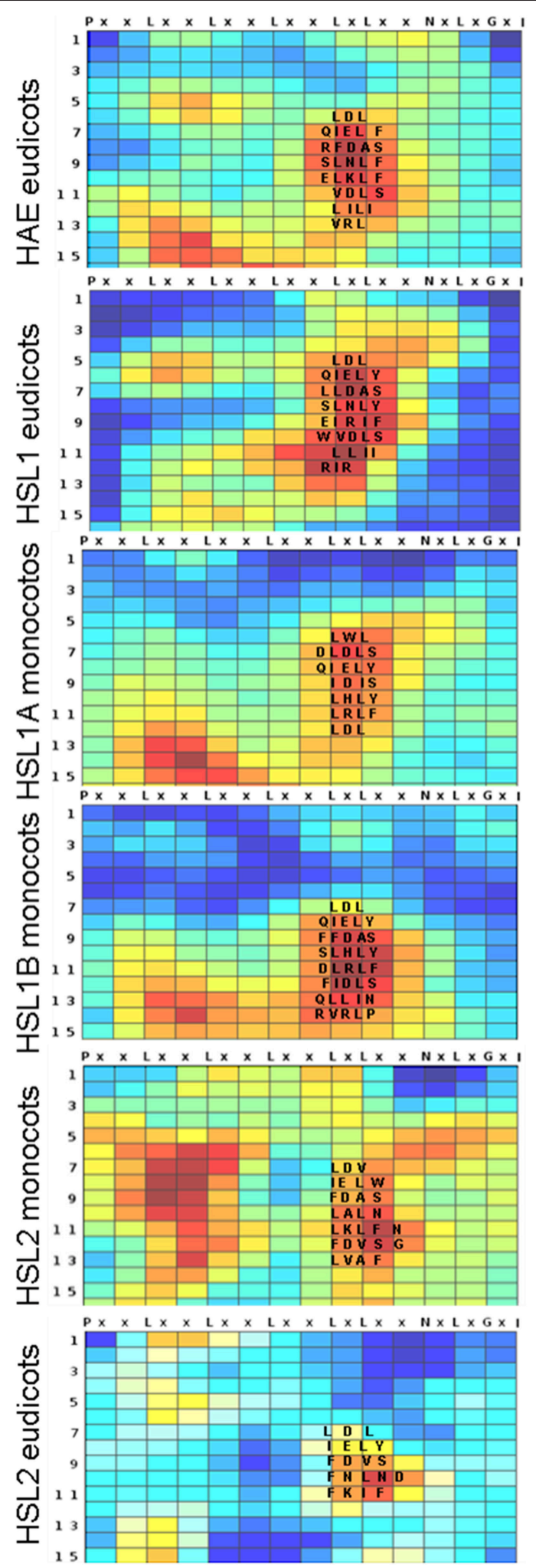

FIGURE 2 | Conserved amino acids in the LRR of HSL proteins. Heatmaps generated using Repeat Conservation Mapping (RCM) of LRR domains (http://144.92.198.58/main/main.php) reflecting degree of identity

(Continued)

\section{FIGURE 2 | Continued}

and similarity of aa residues in a given position ( $X$ axis) in a given repeat ( $Y$-axis) on the surface of the LRR domain of HAE orthologs from the eudicots, HSL1 from eudicots, HSL1A and B from monocots, HSL2 from eudicot and from monocots. The heatmaps are generated using the same ortholog sequences as used for the phylogenetic analysis (Supplementary Figure S1). The alignments used for heatmap construction are available as Supplementary Data Sheet 2.

(His, H) asparagine (Asn, N) at the end are found in all $A$. thaliana IDA/IDL peptide sequences. Experiments where the part of the AtIDA gene encoding PIP was swapped with the corresponding AtIDL sequence and the recombinant AtIDA-IDL gene introduced in the ida mutant, indicate the importance of different aa residues (Stenvik et al., 2008). The AtIDL1 peptide (LVPPSGPSMRHN) complements the mutation, suggesting that the initial Pro is of little importance, that the hydrophobic isoleucine (Ile, I) and the small central Ala residues can be exchanged with the hydrophobic valine (Val, V) and the small Gly, respectively, without affecting the biological activity, and furthermore that a positively charged aa in position 9 is no absolute requirement. In contrast, AtIDL2, AtIDL3, AtIDL4, and AtIDL5 cannot fully complement the ida mutation (Stenvik et al., 2008). Their PIP motifs are characterized by arginine-lysine (ArgLys, RK) in position 9 and 10, in contrast to the LysArg (KR) found in mIDA.

BLAST and tBLASTn searches were executed using the Cterminal sequences of $A$. thaliana IDA and IDL (i.e., PIP extended with eight aa N-terminally, and the C-terminal aa, Figure 3A) BLAST hits were curated for length of the coding region $(<120$ aa), presence of hydrophobic secretion signal, the C-terminal position of the conserved PIP motif, and the presence of the conserved Pro, Ser, and HisAsn residues. IDA and/or IDL sequences were identified in all groups in our data sets.

We were unable to infer a phylogeny over the evolution of IDA, due to the short sequence of the conserved peptide. IDA lacks phylogenetic information and is also present in a high number of copies in many species; any possible pattern was unclear, with no clear clades, branching patterns, and a general lack of stability. Despite this we attempted an inference based on multiple datasets (PIP, EPIP, and EPIP$\mathrm{C}$, Figure 3A), considering species that have undergone fewer genome duplications (Vanneste et al., 2014), however, without success.

As an alternative approach to understand the evolution of IDA/IDL peptides, we grouped the different PIP motifs of the BLAST-identified proteins based on their amino acid composition. In all eudicots, peptides could be classified either as IDA/IDL1 ([P,L][V,I]PPS[A,G]PSKRHN) or like IDL2-5 (PIP[A,T,H,P]S[A,G]PSRKHN), named PIP R $_{\text {and } \text { PIP }_{K}}$ respectively based on the residue in position 10 (Figures $3 B, C$ ). Both motifs were found in every eudicot species tested. In the monocot dataset $\mathrm{PIP}_{\mathrm{R}}$ was found in the orders Arecales and Zingiberales. Additionally, we identified a version in all monocots with close resemblance to $\mathrm{PIP}_{\mathrm{R}}$, but containing a glutamine $(\mathrm{Gln}, \mathrm{Q})$ in position $10\left(\mathrm{PIP}_{\mathrm{Q}}\right.$, Figure $\left.3 \mathrm{D}\right)$. 


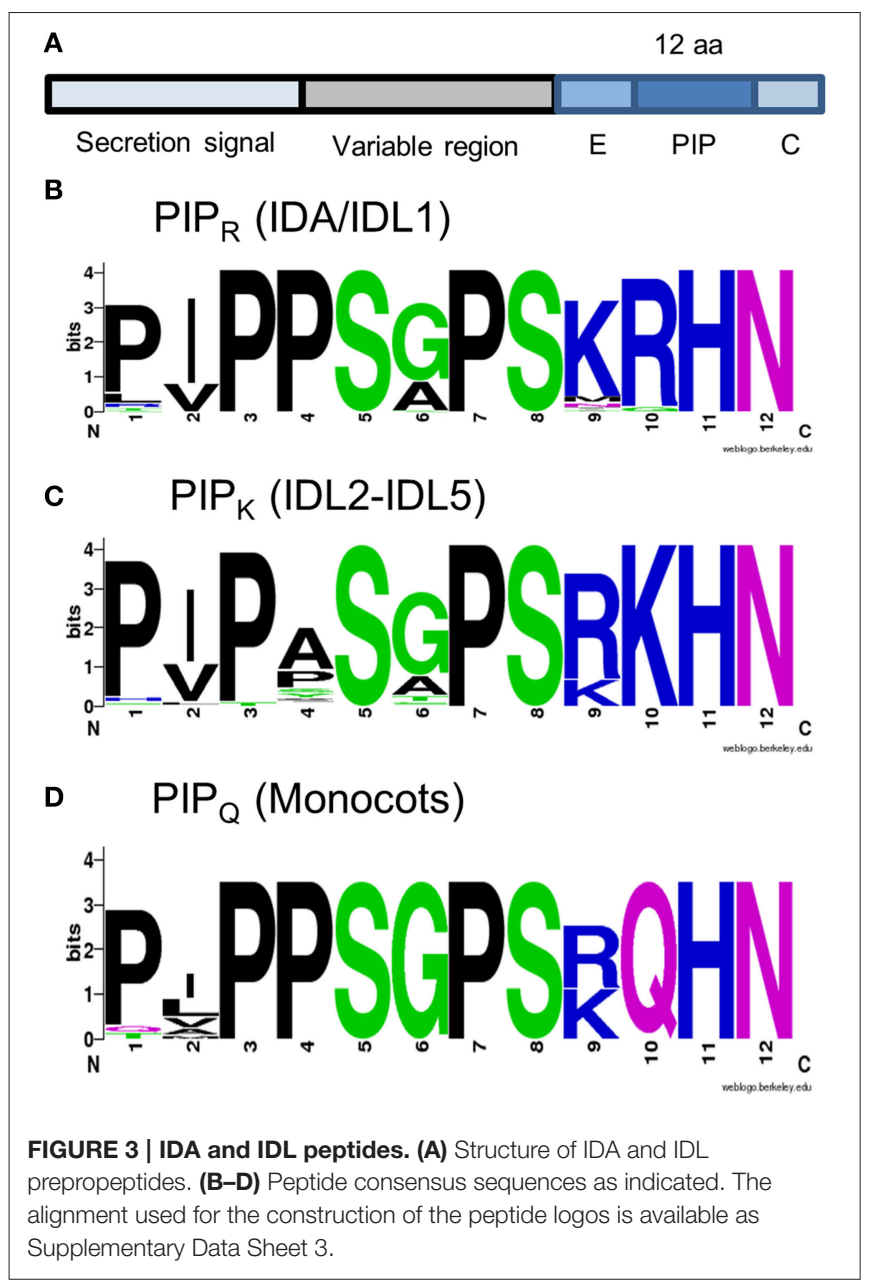

\section{The IDL Gene Family has Expanded in Many Taxa of Core Eudicots}

About 65 million years (Myr) ago after the split of the core eudicots into Rosids and Asterids, whole genome duplication (WGD) took place in a number of eudicot lineages and expanded both the number of species and the gene number of each species (Van de Peer et al., 2009). In line with this, detailed inspection suggests independent evolution especially of the IDL2-5 genes in these two clades. Soybean (Glycine max), common bean (Phaseolus vulgaris) and A. thaliana belong to the Rosids, the beans representing the families of legumes (Fabales), and A. thaliana belonging to the Brassicales. Soybean has six pairs of IDL genes, each pair represented only once in common bean, consistent with a late genome duplication in soybean after the separation from bean (Tucker and Yang, 2012). Tomato (Solanum lycopersicum), potato (Solanum tuberosum), and tobacco (Nicotiana ssp.) belong to the family Solanales in the Asterid clade, and are considered diploid species. However, analyses of inter- and intrachromosomal duplications in the tomato and potato genomes suggest a second more recent WGD in the common ancestor of Petunoideae, Nicotianoideae, and Solanoideae (Song et al., 2012). We have found pairs of almost identical IDL genes both in $N$. benthamiana and tomato that are more similar within than between the species (Supplementary Figure S3A), suggesting that additional independent duplications have taken place after the divergence of Nicotianoideae and Solanoideae. Similarly, we have previously suggested that the AtIDL2 and AtIDL3 genes in A. thaliana result from a recent duplication event (Stenvik et al., 2008).

\section{IDA Ligands and HSL Receptors are Expressed in a Variety of AZs and Species}

Having identified orthologs of IDA and its receptors in a variety of species, we examined in the literature and experimentally whether IDA/IDL1 and the relevant receptors were likely to be involved in cell separation events.

\section{Floral Organ and Flower Abscission}

Abscission plays an important role during plant reproduction. It is common among eudicots (including $A$. thaliana) to abscise turgid floral organs after pollination, when they no longer are functional (van Doorn, 2001). In some species the function of organs change during development, exemplified by tepals in Eriophyllum spp. (Asterales) and Polygonum spp. (Caryophyllales) that first act as protection of reproductive organs and later as organs involved in seed dispersal. In monocots floral organs may wither without abscission (van Doorn, 2001, 2002), or the perianth is retained and will cover the fruits during seed maturation. Hence, in such settings IDA and its receptors are not expected to be active. We postulate that the signaling system is intact in monocots in species where abscission of individual organs takes place when the tepals are free, like in tulips (Tulipa spp., Liliales). Unfortunately genomic and transcriptomic data are scarce for many monocots, especially the Liliales which have gigantic genomes (Shahin et al., 2012).

Normally A. thaliana does not shed cauline leaves, whole flowers or fruits. However, ectopic expression of IDA and IDL peptides in $A$. thaliana leads to induction of abscission in a HAE/HSL2-dependent manner at the base of pedicels, cauline leaves, and inflorescence branches, rather than a general separation of cells in the plant (Figures 4A-D) (Stenvik et al., 2006). These are sites of abscission in other species. Interestingly, A. thaliana lines transformed with promoter:GUS constructs for HAE or HSL2 show that the receptor genes are expressed in the vestigial AZs at these sites (Figures 4E-G).

Fall of whole flowers is less common than floral organ abscission (Figure 5A), but occurs in both monocot and eudicot species, when pollination or fertilization fail (van Doorn, 2002). Abscission of immature fruit is a normal event in several cultivated species. In Citrus spp. (Sapindales), abscission of flowers and young fruit results from cell separation at an AZ at the base of the floral pedicel. The CicIDA3 gene, expressed in Citrus clementina AZs, encodes a protein with a PIP motif identical to mIDA with the exception of a Gly instead of an Ala in position 6 (Estornell et al., submitted). Overexpression of CicIDA3 in A. thaliana induced the same phenotypic changes as have been shown for overexpression of IDA and AtIDL1 (Figures 4A-D) (Estornell et al., submitted), suggesting that the 


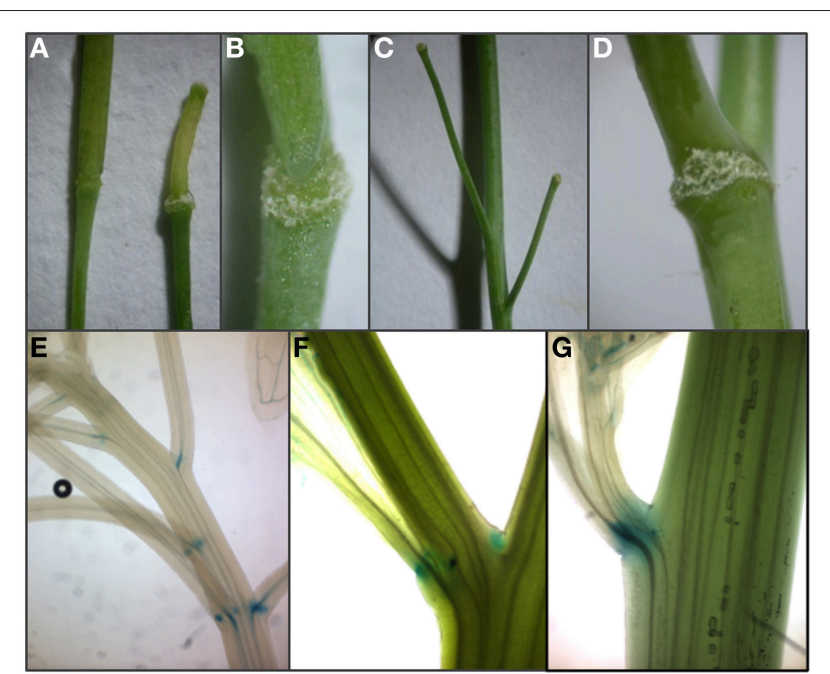

FIGURE 4 | Expression of HAE and HSL2 promoter: GUS constructs at sites of ectopic abscission. (A-C) Enlargement of AZ and premature abscission of whole flowers and immature fruits compared to wild type silique (to the left in $\mathbf{A}$ ) in A. thaliana plants overexpressing AtIDL1. (D) Enlarged vestigial AZ after abscission of a cauline leaf in A. thaliana overexpressing AtIDL1. (E,F) pHAE:GUS expression and (G) pHSL2:GUS expression in vestigial AZs at the bases of pedicels, branches, and cauline leaves.

citrus prepropeptide undergo the correct processing, that the Citrus peptide can activate IDA's receptors and thus provides experimental evidence supporting a function in citrus abscission events.

\section{Leaf Abscission}

AZs, with layers of rather undifferentiated small cells, are normally formed during organ development both in A. thaliana and other species. Additionally, ethylene may promote the formation of new AZs and thereby induce abscission (Roberts et al., 2000). A thorough investigation of IDA and HAE/HSL2 in connection with ethylene-induced leaf abscission has been performed in soybean (Glycine max), common bean and tomato (Tucker and Yang, 2012). Of the five genes encoding tomato IDA/IDL peptides, one with a PIP motif identical to that of mIDA was expressed in ethylene-induced AZs on leaf pedicels. Likewise, the GmIDA2a and 2b, which had the highest expression level in leaf $A Z$ and the highest relative expression ratio between $\mathrm{AZ}$ and petiole tissue both in the presence and absence of ethylene, are the paralogs most similar to AtIDA, which also display ethylene independent expression. The GmHAE3b and GmHAE5a/5b with the highest similarities to A. thaliana HAE and HSL2, respectively, were also adequately expressed in the petiole (Tucker and Yang, 2013). Thus, these expression patterns support the involvement of the IDA-HAE/HSL2 module in leaf abscission both in Rosids and Asterids.

One of the most visible of all shedding processes in angiosperms is the leaf fall (Figure 5B) at the beginning of the dormant (i.e., cold or dry) season in deciduous trees, triggered by seasonal reduction of the photoperiod and temperature (Taylor and Whitelaw, 2001; Keskitalo et al., 2005). Autumnal

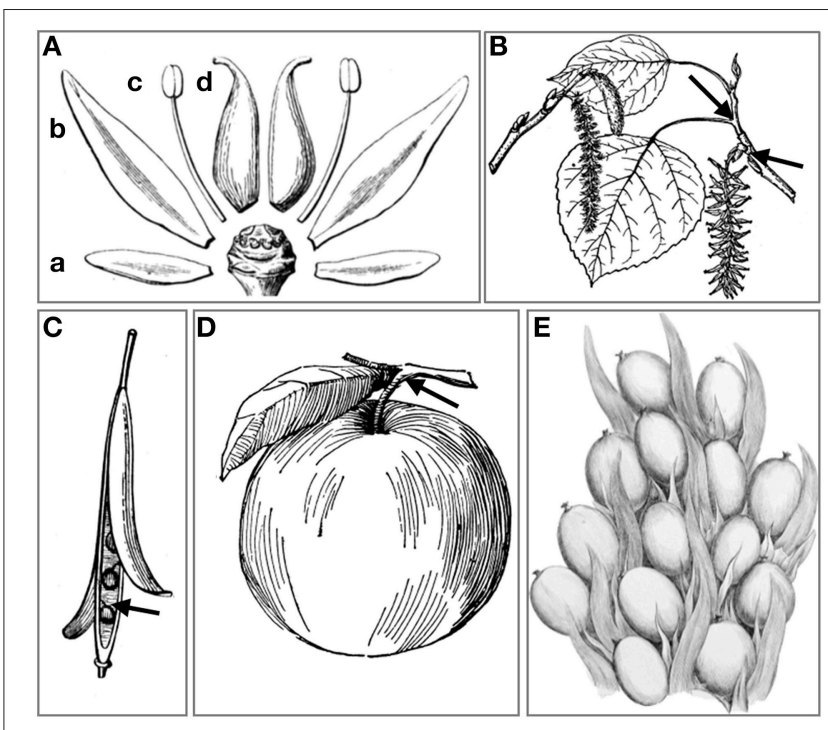

FIGURE 5 | Modes of abscission. (A) Abscission of (a) sepals, (b) petals, (c) stamen, and (d) carpels. (B) Abscission of leaves at the axil of the pedicel, and abscission of entire male inflorescence (catkin) in Populus spp. (C) Opening of valves in dehiscence zones of dry many-seeded capsules, and abscission of individual seeds. (D) Abscission of fleshy fruits at AZ on pedicel. (E) The oil palm drupe fruit are tightly arranged within spikelets and abscise one by one when ripe. (A-D) Image courtesy the private collection of Roy Winkelman. First published in Gray (1858) and Foster (1921). (E) Image courtesy Missouri Botanical Garden. http://www.botanicus.org.

leaf abscission provides deciduous trees with resistance against drought and freezing damage (Fischer and Polle, 2010; Zanne et al., 2014). Species with N-fixing symbionts like alder (Alnus spp., Fagales) can afford to lose green leaves, while other deciduous trees, like Populus ssp. (Malpighiales), withdraw elements of valuable molecules (as chlorophyll) and shed yellowish or reddish leaves (Keskitalo et al., 2005; Fracheboud et al., 2009). Hence, the senescence process that allows trees to conserve resources and prepare for a dormant period terminates in a cell separation event in preformed AZs at the base of the pedicels.

Leaf abscission can be induced in hybrid aspen (Populus tremula X P. tremuloides) by depriving the leaves of light (Jin et al., 2015). In our experimental setup, $50 \%$ of the shaded leaves separated from the branch at the axil after 8 days, while non-shaded leaves were not shed. We designed gene-specific primers against the IDA,IDL1, and HAE orthologs of Populus ssp., which seem to have lost the HSL2 ortholog. The two genes most similar to AtIDA were significantly upregulated in the axil upon induction of leaf abscission (Figure 6A). As in soybean, the expression levels of the receptors did not change in the course of this experiment, suggesting that the timing of abscission is rather dependent on the induction of IDA peptides than on the transcriptional regulation of the receptor.

\section{Seed and Fruit Abscission}

The fertilized ovule of Angiosperms (the seed) and surrounding maternal tissue that together constitutes the fruit, needs to be separated from the mother plant in one way or another when 

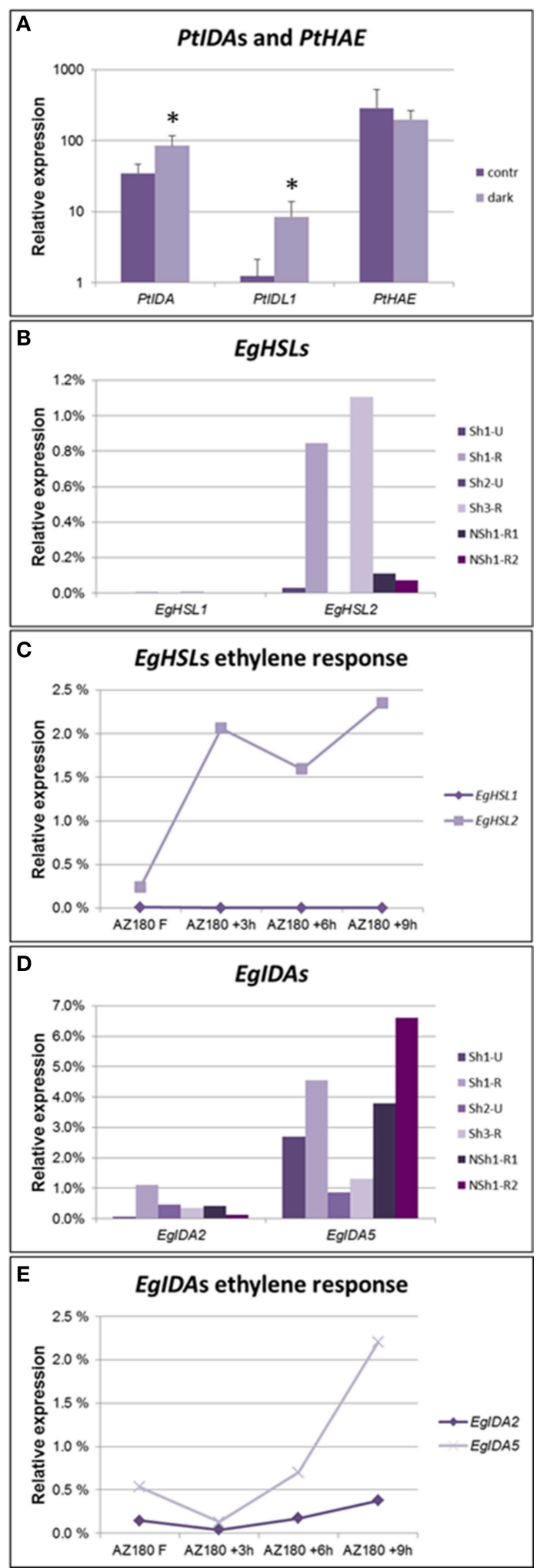

FIGURE 6 | Expression levels of genes encoding IDA ligands and HSL receptors in AZs. (A) qPCR detecting increased expression levels of Populus IDA genes, but not the PIHAE gene in axils of shade-treated leaves prone to

(Continued)

\section{FIGURE 6 | Continued}

abscise compared to axils in non-shaded, non-abscission aspen leaves. qPCR, averages and standard deviations of three biological replicates. Normalized to PtACTIN1 expression. * $p<0.05, t$-test, non-shaded vs. shaded. (B) qPCR analysis of oil palm EgHSL1 and EgHSL2 expression in AZs from unripe (Sh1-U and Sh2-U) not actively abscising fruit and AZs of ripe (Sh1-R, Sh3-R) actively abscising fruit, as well as AZs from ripe fruit of a non-abscising tree (NSh1-R1, NSh1-R2). (C) qPCR analyses of oil palm EgHSL expression during ethylene-induced abscission in ripe 180 DAP fruits. Samples were taken after 0, 3, 6, and $9 \mathrm{~h}$ treatment with ethylene. Similar results were obtained when treating 145 DAP fruits (Supplementary Figure S3C). In both experiments fruit separated by $9 \mathrm{~h}$ of ethylene treatment. (D) qPCR analysis of oil palm EgIDA2 and EgIDA5 expression in the AZs of unripe (Sh1-U and Sh2-U) not actively abscising fruit and AZs of ripe (Sh1-R, Sh3-R) actively abscising fruit, as well as AZs from ripe fruit of a non-abscising tree (NSh1-R1, NSh1-R2). Expression levels of additional EgIDA genes are found in Supplementary Figure S3D. (E) qPCR analyses of oil palm EgIDA2 and EgIDA5 expression during ethylene-induced abscission in ripe 180 DAP fruits. Samples were taken after $0,3,6$, and $9 \mathrm{~h}$ treatment with ethylene. Similar results were obtained when treating 145 DAP fruits (Supplementary Figure S3E). In both experiments fruit separated by $9 \mathrm{~h}$ of ethylene treatment.

ripe. In botanical terms there are roughly four main types of fruits: berries (fleshy most often many-seeded fruits), drupes (fleshy most often one-seeded fruits with hard exocarp), capsules, including siliques and pods (dry many-seeded fruits), and nuts, including caryopses of grasses (dry one-seeded fruits). Nuts are shed and abscised at the base of the carpels, while many-seeded capsules or pods open along dehiscence zones (Figure 5C). This is seen in a wide range of angiosperms such as orchids (Asparagales), poppies (Papaver spp., Ranunculales), and tobacco (Nicotiana tabacum, Solanales) as well as A. thaliana, before each single seed abscise where the funiculus is attached to the seed. We have earlier reported that AtIDA and HAE are expressed in A. thaliana dehiscence zones (Butenko et al., 2006).

For fleshy fruit eaten directly from the plant by birds or animals e.g., blueberries (Vaccinium myrtillus, Ericales), abscission is not necessitated for seed dispersal. Nevertheless, both larger fruits like apples (Malus domestica, Rosales) (Figure 5D) and drupes like olives (Olea europaea, Asterides) (Gil-Amado and Gomez-Jimenez, 2013) may abscise at the apex or at the base of the pedicel (fruit stalk), so that animals may pick up the fruits from the ground. In line with this, HAE was recently shown to be strongly upregulated in abscising olives compared to the preabscising AZs (Gil-Amado and Gomez-Jimenez, 2013).

To test whether IDA-HAE/HSL2 is involved in control of fruit abscission also in monocots we chose to investigate abscission of the fruits of oil palm (Elaeis guineensis, Arecales). The flowers of the oil palm grow in large clusters giving rise to plumb-sized reddish fruits in large bunches (Figure 5E) that have large, preformed, multilayered AZs at the boundary of the mesocarp and the pedicel. Under natural conditions it takes about 160 days from pollination until the fruits are ripe and start separating from the bunches on the trees. mRNA was isolated from AZs of unripe and ripe fruit of trees that abscise their fruit (Shedding-Sh1, Sh2, and Sh3), and additionally from AZs of an unusual tree (Non-shedding, NSh1) that fails to abscise its fruit. We identified genes encoding HSL1 and HSL2 
receptors; EgHSL1 had a very low expression level that did not change over time (Figure 6B). EgHSL2 on the other hand was low in unripe AZs of shedding trees, as well as AZs of ripe NSh1 fruit, but increased strongly in AZs of ripe fruit of abscising trees. From about 145 DAP, ethylene treatment induce fruit abscission after $9 \mathrm{~h}$. This treatment also induces increased EgHSL2 expression (Figures 6C, Supplementary Figure S3C). Thus, EgHSL2 expression was consistently associated with active fruit abscission.

We identified $10 I D A$ oil palm genes that come in five pairs encoding almost identical preproproteins (Supplementary Figure $\mathrm{S} 3 \mathrm{~B})$, suggesting recent genome duplication. Expression of five of these genes (EgIDA2 to EgIDA6) was detected in AZs of E. guineensis fruits using RT qPCR (Supplementary Figure S3D). In trees showing fruit abscission EgIDA2, EgIDA4 and the most abundant, EgIDA5, all had their highest expression levels when fruit are separating (Figure 6D), and EgIDA2 and EgIDA5 could also be induced by ethylene (Figure 6E). These genes encode PIPs near to identical with mIDA; like CicIDA3, EgIDA5 differs only by an exchange of the small Ala with the small Gly in position 6 . Interestingly, the EgIDA genes were not strictly associated with separation; as transcripts were present also in ripe AZs of the non-shedding tree (Figure 6C). Hence, EgIDA are expressed and induced in NSh1 although the receptor gene EgHSL2 has a very low level of expression.

\section{DISCUSSION}

Our phylogenetic analyses of the HSL LRR-RLKs have revealed that the evolution of this receptor family is congruent with that of angiosperm species evolution, and makes it likely that the common ancestor of angiosperms had both a HSL1 and a HSL2 gene (Figure 7). Both receptors are present already in the basal angiosperm (e.g., Amborella), where stamen abscission is a common feature that facilitates the access of pollinators (beetles and flies bringing pollen from other flowers) to the female organ (Endress, 2010). The IDA gene with $\mathrm{PIP}_{\mathrm{R}}$ motif identified in Amborella, may play a role here, similar to IDA's role as a signaling ligand in sepal, petal, and stamen abscission in $A$. thaliana (Butenko et al., 2003, 2014).

While the receptors follow the general evolution of the angiosperms, the PIP $_{R}$ motif likely to represent the peptide released from the propeptide upon secretion, has virtually been preserved without substantial changes throughout the evolution of flowering plants. In a short peptide that presumably shall bind to given aa side chains in the receptor, there are limited possibilities for aa changes that retain the relevant binding properties: Exchange of one small aa for another might pass, but exchange of an aa with different size or charge of the side chain (like a Lys or a Asn for an Arg), may affect the structure of the ligand and its binding properties (Czyzewicz et al., 2015).

The genome of a common ancestor of flowering plants has been suggested to harbor between 10,000 and 14,000 genes (Tang et al., 2008) while a genome size of 25,000-50,000 genes is common in modern angiosperms. This increase is due to a number of WGD, including a triplication in dicots before the separation of the Vitis. Two additional duplications and a subsequent extensive loss of duplicated genes occurred in $A$. thaliana (Tang et al., 2008; Van de Peer et al., 2009). Relevant for our analyses is that HSL1 has been duplicated in two separate events: Firstly, before the split of Vitales, Asterids, and Rosids, within eudicots to form $H A E$, and secondly, within monocots to form a more recent duplication before the split of Arecales. Still, multiple synteny investigations between five different and partly distantly related species (Arabidopsis, Carica, Populus, Vitis, and Oryza) showed that a total of $61 \%$ of the A. thaliana genes had preserved their ancestral locations (Tang et al., 2008). There are controversies regarding the placement of the smaller clades of Ceratophyllales and Caryophyllales, as well as Vitales, in relation to the eudicots (Zeng et al., 2014). Our analyses of the three receptor genes support Vitales and Caryophyllales as sister groups of the core eudicots. HSL1/HAE duplication is found on syntenic genome regions in the other eudicots, but not rice, supporting that $H A E$ was generated by a WGD event (Tang et al., 2008). Specific genomic locations of AtIDA and HSL2 and their neighboring genes in A. thaliana are preserved in Populus and Vitis, respectively (Woodhouse et al., 2011). The IDL4 is positioned in synteny with other eudicots, but the other $I D L$ genes may rather be a result of duplications known to have taken place after the divergence of Brassicaceae and Caricaceae families (Tang et al., 2008).

Duplications provide the opportunity for the new copy to evolve, like giving rise to the $\mathrm{PIP}_{\mathrm{K}}$ or $\mathrm{PIP}_{\mathrm{Q}}$ motif varieties. Transition from Arg to Lys or Gln may only require one nucleotide change. Based on available sequence data we hypothesize that IDL4 with PIP $_{K}$ evolved before the split of Proteales, however this variant may have a more basal history within angiosperm evolution. Likewise, the $\mathrm{PIP}_{\mathrm{Q}}$ version has presumably developed after genome duplication in the monocot lineage, and we hypothesize that $\mathrm{PIP}_{\mathrm{Q}}$ is present in all monocot orders (Figure 7). Sequence data for Acorales is needed to substantiate this. PIP $P_{R}$ may represent the original peptide gene that has been lost from some of the monocot orders: Alismatales and Poales are devoid of the gene, whilst it is present in Arecales and Zingiberales, suggesting independent loss for some lineages. This is more parsimonious than a basal loss within the monocots followed by multiple gains. A final conclusion must await genomic sequencing of species from orders where data at present are lacking.

We have evidence from $A$. thaliana that IDA can be substituted with AtIDL1, but the other AtIDL genes only to a limited extent can substitute for IDA function, indicating that their encoded peptides do not interact properly with the HAE and HSL2 receptors (Stenvik et al., 2008). The high similarity between conserved aa in the ectodomain as well as the kinase domain of HSL1 compared to HAE, might suggest HSL1 to be their native receptor. This would require closely related expression patterns for HSL1 and IDL genes. However, at least in Arabidopsis, the expression patterns of HSL1 and these IDL genes do not suggest involvement in floral organ abscission (Cho et al., 2008; Stenvik et al., 2008), and mutant phenotypes are so far lacking. Different methods have lately been suggested for testing of peptide-ligand receptor interactions (Butenko et al., 2009, 2012, 2014). Interaction of the mIDA 


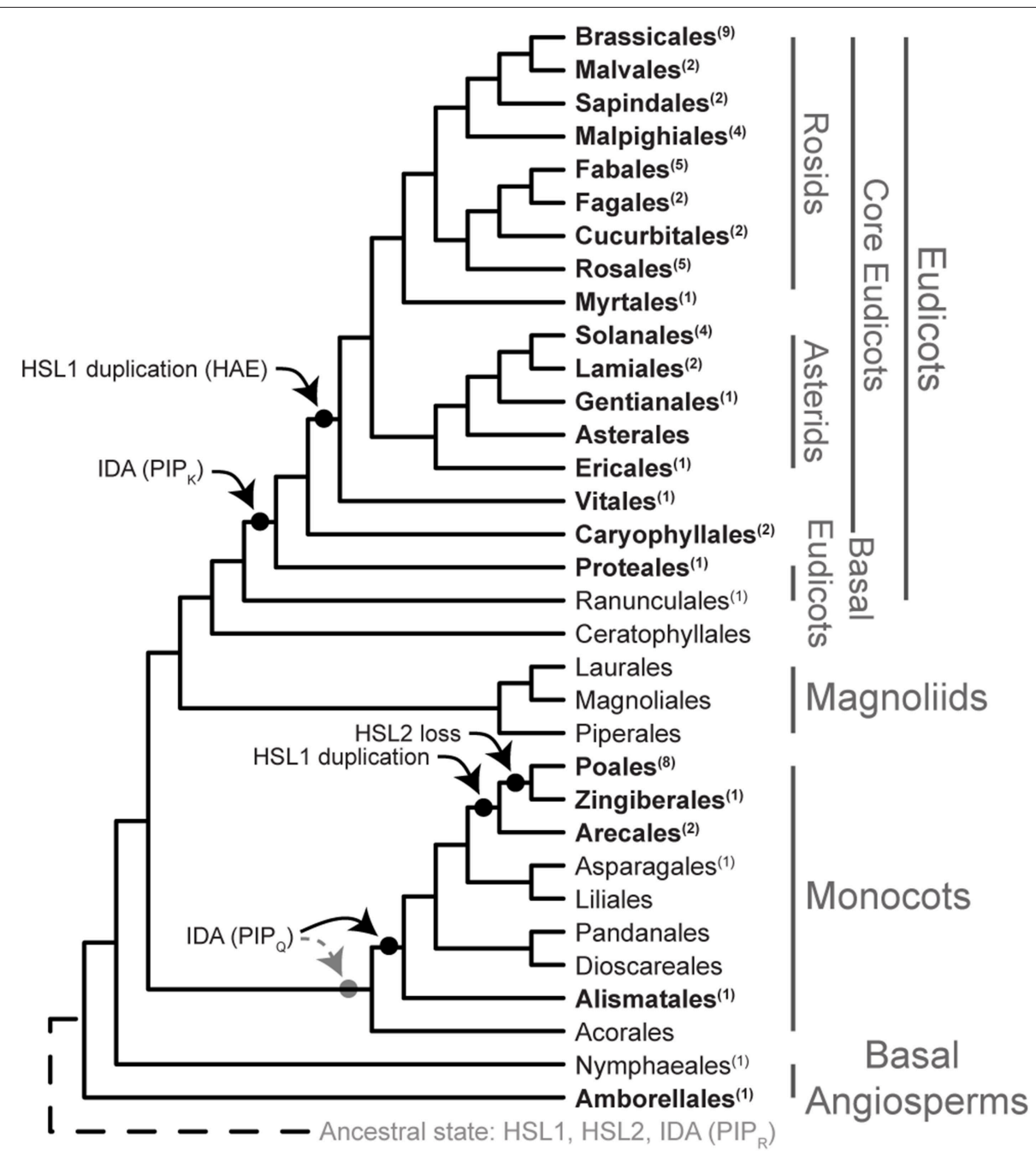

FIGURE 7 | Evolution of the IDA HAE/HSL2 signaling module. Phylogeny of Angiosperms adapted from Zhang et al. (2012), Vanneste et al. (2014), Zeng et al. (2014), and Dohm et al. (2014). Taxonomical levels are taken from Zeng et al. (2014). Numbers in superscript behind order names represent the number of species used in the analysis. Order names in bold represent those orders with at least one completely sequenced genome. The two possible evolutionary origins of IDA (PIP $\mathrm{Q}$ ) in monocots are illustrated.

peptide with HSL2 expressed in N. benthamiana, results in an immediate oxidative burst, similar to elicited LRR-RLKs involved in pathogen defense (Butenko et al., 2014). A thousand times more peptide is needed to provoke such a reaction from the HAE receptor under the same conditions, although the two receptors in genetic terms are functionally redundant with respect to control of floral organ abscission (Cho et al., 2008; Butenko et al., 2014). We have found distinct evolutionarily conserved differences between the kinase domains of HAE/HSL1 and that of HSL2. This may explain the differences in readout between HAE and HSL2 in the presence of IDA or IDL peptides (Butenko et al., 2014).

Interestingly, within monocots HSL1 has been duplicated before the split-off of the Arecales, and HSL2 has been lost after the split of Arecales, leaving the orders Poales, and Zingiberales devoid of the gene. Abscission still takes place in these orders, e.g., "finger drop" in banana (Musa spp.) and caryopsis shedding (diminished in the domesticated species) in the grasses. We have demonstrated that oil palm of the Arecales use HSL2 and IDA genes encoding $\mathrm{PIP}_{\mathrm{R}}$ peptides in connection to fruit abscission. It is tempting to suggest that the $\mathrm{PIP}_{\mathrm{Q}}$ version of the IDA peptide have developed to fit one or the other HSL1 receptors.

We have presented examples of the presence of IDA and its receptors in all the major groups of Angiosperms during abscission of floral organs, flowers, immature, and mature fruits and of leaves. The first Angiosperm developed more than 150 Myr ago, and IDA ligands may have been conserved most of this time. At the same time the number of IDA and receptor genes has increased and a variety of modes of organ detachment has developed. This lends support to the notion that it is not what you do, but where and when you do it that is important (Carroll, 2008). New gene copies have presumably made it possible to change place and time for cell separation events. 


\section{AUTHOR CONTRIBUTIONS}

IS, RO, KF, XJ, JK, and IN performed the research; IS, RO, UF, TT, and RA analyzed the data; IN and RA designed the research, RA wrote the article with contributions from IS, RO, UF, TT, and IN.

\section{FUNDING}

This research was supported by grant no 213785 from the Research Council of Norway to RA; by Deutsche Forschungsgemeinschaft (DFG, Fi1661/1-1) to UF; to XJ by Bio4Energy, the Berzelii Centre (Vinnova) and Stiftelsen Mauritz

\section{REFERENCES}

Aalen, R. B. (2011). "Flower and floral organ abscission - control, gene expression and hormone interaction," in The Flowering Process and its Control in Plants: Gene Expression and Hormone Interaction, ed M. W. Yaish (Trivandrum: Research Signpost/Transworld Research Network), 307-327.

Aalen, R. B., Wildhagen, M., Stø, I. M., and Butenko, M. A. (2013). IDA: a peptide ligand regulating cell separation processes in Arabidopsis. J. Exp. Bot. 64, 5253-5261. doi: $10.1093 /$ jxb/ert338

Abascal, F., Zardoya, R., and Posada, D. (2005). ProtTest: selection of best-fit models of protein evolution. Bioinformatics 21, 2104-2105. doi: 10.1093/bioinformatics/bti263

Bleecker, A. B., and Patterson, S. E. (1997). Last exit: senescence, abscission, and meristem arrest in Arabidopsis. Plant Cell 9, 1169-1179. doi: 10.1105/tpc.9.7.1169

Butenko, M. A., Albert, M., and Aalen, R. B. (2012). "Methods to identify new partners of plant signalling peptides," in Plant Signaling Peptides, eds H. R. Irving and C. Gehring (Berlin; Heidelberg: Springer-Verlag), 241-256.

Butenko, M. A., Patterson, S. E., Grini, P. E., Stenvik, G. E., Amundsen, S. S., Mandal, A., et al. (2003). Inflorescence deficient in abscission controls floral organ abscission in Arabidopsis and identifies a novel family of putative ligands in plants. Plant Cell 15, 2296-2307. doi: 10.1105/tpc.014365

Butenko, M. A., Stenvik, G.-E., Alm, V., Sæther, B., Patterson, S. E., and Aalen, R. B. (2006). Ethylene-dependent and -independent pathways controlling floral abscission are revealed to converge using promoter::reporter gene constructs in the ida abscission mutant. J. Exp. Bot. 57, 3627-3637. doi: 10.1093/jxb/erl130

Butenko, M. A., Vie, A. K., Brembu, T., Aalen, R. B., and Bones, A. M. (2009). Plant peptides in signalling: looking for new partners. Trends Plant Sci. 14, 255-263. doi: 10.1016/j.tplants.2009.02.002

Butenko, M. A., Wildhagen, M., Albert, M., Jehle, A., Kalbacher, H., Aalen, R. B., et al. (2014). Tools and strategies to match peptide-ligand receptor pairs. Plant Cell 26, 1838-1847. doi: 10.1105/tpc.113.120071

Carroll, S. B. (2008). Evo-Devo and an expanding evolutionary synthesis: a genetic theory of morphological evolution. Cell 134, 25-36. doi: 10.1016/j.cell.2008.06.030

Castresana, J. (2000). Selection of conserved blocks from multiple alignments for their use in phylogenetic analysis. Mol. Biol. Evol. 17, 540-552. doi: 10.1093/oxfordjournals.molbev.a026334

Cho, H.-T., and Cosgrove, D. (2000). Altered expression of expansin modulates leaf growth and pedicel abscission in Arabidopsis thaliana. Proc. Natl. Acad. Sci. U.S.A. 97, 9783-9788. doi: 10.1073/pnas.160276997

Cho, S. K., Larue, C. T., Chevalier, D., Wang, H., Jinn, T. L., Zhang, S., et al. (2008). Regulation of floral organ abscission in Arabidopsis thaliana. Proc. Natl. Acad. Sci. U.S.A. 105, 15629-15634. doi: 10.1073/pnas.0805539105

Curtis, M. D., and Grossniklaus, U. (2003). A gateway cloning vector set for highthroughput functional analysis of genes in planta. Plant Physiol. 133, 462-469. doi: 10.1104/pp.103.027979

Czyzewicz, N., Wildhagen, M., Cattaneo, P., Stahl, Y., Pinto, K. G., Aalen, R. B., et al. (2015). Antagonistic peptide technology for functional dissection
Carlgrens Fond; and by Franco-Thai and Thailand Graduate Institute of Science and Technology (TGIST) scholarships to KF, and funding from PalmElit SAS (KF, TT).

\section{ACKNOWLEDGMENTS}

We thank Nora M. Tandstad for the images of 35S:IDL1 plants, and Even Sannes Riiser for the pHAE:GUS and pHSL2:GUS reporter lines. We thank Anek Limsrivilai at GoldenTenera Oil Palm Plantation in Thailand and Roberto Poveda at the PDA/Murrin Company in Quinindé, Ecuador for the oil palm material. We appreciate the technical support from Julien Serret for the oil palm qPCR analysis.

of CLE peptides revisited. J. Exp. Bot. 66, 5367-5374. doi: 10.1093/jxb/ erv284

Dohm, J. C., Minoche, A. E., Holtgräwe, D., Capella-Gutiérrez, S., Zakrzewski, F., Tafer, H., et al. (2014). The genome of the recently domesticated crop plant sugar beet (Beta vulgaris). Nature 505, 546-549. doi: 10.1038/nature 12817

Durek, P., Schmidt, R., Heazlewood, J. L., Jones, A., Maclean, D., Nagel, A., et al. (2010). PhosPhAt: the Arabidopsis thaliana phosphorylation site database. An update. Nucleic Acids Res. 38, D828-D834. doi: 10.1093/nar/gkp810

Endress, P. K. (2010). The evolution of floral biology in basal angiosperms. Philos. Trans. R. Soc. Lond. B Biol. Sci. 365, 411-421. doi: 10.1098/rstb.20 09.0228

Fischer, U., and Polle, A. (2010). "Populus responses to abiotic stress," in Genetics and Genomics of Populus, eds S. Jansson, R. Bhalerao, and A. Groover (New York, NY: Springer), 225-246.

Fracheboud, Y., Luquez, V., Björken, L., Sjödin, A., Tuominen, H., and Jansson, S. (2009). The control of autumn senescence in European aspen. Plant Physiol. 149, 1982-1991. doi: 10.1104/pp.108.133249

Foster, E. D. (1921). The American Educator. Vol. 1, Chicago, IL: Ralph Durham Company.

Gil-Amado, J. A., and Gomez-Jimenez, M. C. (2013). Transcriptome analysis of mature fruit abscission control in olive. Plant Cell Physiol. 54, 244-269. doi: $10.1093 / \mathrm{pcp} / \mathrm{pcs} 179$

González-Carranza, Z. H., Elliott, K. A., and Roberts, J. A. (2007). Expression of polygalacturonases and evidence to support their role during cell separation processes in Arabidopsis thaliana. J. Exp. Bot. 58, 3719-3730. doi: $10.1093 / \mathrm{jxb} / \mathrm{erm} 222$

Gray, A. (1858). How Plants Grow; A Simple Introduction to Structural Botany. New York, NY: American Book Company.

Helft, L., Reddy, V., Chen, X., Koller, T., Federici, L., Fernández-Recio, J., et al. (2011). LRR conservation mapping to predict functional sites within protein leucine-rich repeat domains. PLoS ONE 6:e21614. doi: 10.1371/journal.pone.0021614

Jin, X., Zimmermann, J., Polle, A., and Fischer, U. (2015). Auxin is a longrange signal that acts independently of ethylene signaling on leaf abscission in Populus. Front. Plant Sci. 6:634. doi: 10.3389/fpls.2015.00634

Katoh, K., and Toh, H. (2008). Improved accuracy of multiple ncRNA alignment by incorporating structural information into a MAFFT-based framework. BMC Bioinformatics 9:212. doi: 10.1186/1471-2105-9-212

Keskitalo, J., Bergquist, G., Gardeström, P., and Jansson, S. (2005). A cellular timetable of autumn senescence. Plant Physiol. 139, 1635-1648. doi: 10.1104/pp.105.066845

Kumpf, R. P., Shi, C.-L., Larrieu, A., Stø, I. M., Butenko, M. A., Péret, B., et al. (2013). Floral organ abscission peptide IDA and its HAE/HSL2 receptors control cell separation during lateral root emergence. Proc. Natl. Acad. Sci. U.S.A. 110, 5235-5240. doi: 10.1073/pnas. 1210835110

Lashbrook, C. C., and Cai, S. (2008). Cell wall remodeling in Arabidopsis stamen abscission zones: temporal aspects of control inferred from transcriptional profiling. Plant Signal. Behav. 3, 733-736. doi: 10.4161/psb.3.9.6489 
Lewis, M. W., Leslie, M. E., and Liljegren, S. J. (2006). Plant separation: 50 ways to leave your mother. Curr. Opin. Plant Biol. 9, 59-65. doi: 10.1016/j.pbi.2005.11.009

Liu, B., Butenko, M. A., Shi, C.-L., Bolivar, J. L., Winge, P., Stenvik, G.-E., et al. (2013). Nevershed and inflorescence deficient in abscission are differentially required for cell expansion and cell separation during floral organ abscission in Arabidopsis thaliana. J. Exp. Bot. 64, 5345-5357. doi: 10.1093/jxb/ert232

Meir, S., Philosoph-Hadas, S., Sundaresan, S., Selvaraj, K. S. V., Burd, S., Ophir, R., et al. (2010). Microarray analysis of the abscission-related transcriptome in the tomato flower abscission zone in response to auxin depletion. Plant Physiol. 154, 1929-1956. doi: 10.1104/pp.110.160697

Niederhuth, C. E., Patharkar, O. R., and Walker, J. C. (2013). Transcriptional profiling of the Arabidopsis abscission mutant hae hsl2 by RNA-Seq. BMC Genomics 14:37. doi: 10.1186/1471-2164-14-37

Patterson, S. E. (2001). Cutting loose. Abscission and dehiscence in Arabidopsis. Plant Physiol. 126, 494-500. doi: 10.1104/pp.126.2.494

Roberts, J. A., Elliot, K. A., and Gonzalez-Carranza, Z. H. (2002). Abscission, dehiscence and other cell separation processes. Annu. Rev. Plant Biol. 53, 131-158. doi: 10.1146/annurev.arplant.53.092701.180236

Roberts, J. A., Whitelaw, C. A., Gonzalez-Carranza, Z. H., and McManus, M. T. (2000). Cell separation processes in plants- models, mechanisms and manipulation. Ann. Bot. 86, 223-235. doi: 10.1006/anbo.2000.1203

Roongsattham, P., Morcillo, F., Jantasuriyarat, C., Pizot, M., Moussu, S., Jayaweera, D., et al. (2012). Temporal and spatial expression of polygalacturonase gene family members reveals divergent regulation during fleshy fruit ripening and abscission in the monocot species oil palm. BMC Plant Biol. 12:150. doi: 10.1186/1471-2229-12-150

Shahin, A., Van Kaauwen, M., Esselink, D., Bargsten, J., Van Tuyl, J. W., Visser, R. G., et al. (2012). Generation and analysis of expressed sequence tags in the extreme large genomes Lilium and Tulipa. BMC Genomics 13:640. doi: 10.1186/1471-2164-13-640

Shiu, S. H., and Bleecker, A. B. (2001). Receptor-like kinases from Arabidopsis form a monophyletic gene family related to animal receptor kinases. Proc. Natl. Acad. Sci. U.S.A. 98, 10763-10768. doi: 10.1073/pnas.181141598

Song, C., Guo, J., Sun, W., and Wang, Y. (2012). Whole genome duplication of intra- and inter-chromosomes in the tomato genome. J. Genet. Genomics 39, 361-368. doi: 10.1016/j.jgg.2012.06.002

Stamatakis, A. (2006). RAxML-VI-HPC: maximum likelihood-based phylogenetic analyses with thousands of taxa and mixed models. Bioinformatics 22, 2688-2690. doi: 10.1093/bioinformatics/btl446

Stenvik, G.-E., Butenko, M. A., Urbanowicz, B. R., Rose, J. K., and Aalen, R. B. (2006). Overexpression of inflorescence deficient in abscission activates cell separation in vestigial abscission zones in Arabidopsis. Plant Cell 18, 1467-1476. doi: $10.1105 /$ tpc. 106.042036

Stenvik, G.-E., Tandstad, N. M., Guo, Y., Shi, C.-L., Kristiansen, W., Holmgren, A., et al. (2008). The EPIP peptide of inflorescence deficient in abscission is sufficient to induce abscission in Arabidopsis through the receptorlike kinases HAESA and HAESA-LIKE2. Plant Cell 20, 1805-1817. doi: 10.1105/tpc.108.059139

Sun, Y., Li, L., Macho, A. P., Han, Z., Hu, Z., Zipfel, C., et al. (2013). Structural basis for flg22-Induced activation of the Arabidopsis FLS2-BAK1 immune complex. Science 342, 624-628. doi: 10.1126/science.1243825

Tang, H., Wang, X., Bowers, J. E., Ming, R., Alam, M., and Paterson, A. H. (2008). Unraveling ancient hexaploidy through multiply-aligned angiosperm gene maps. Genome Res. 18, 1944-1954. doi: 10.1101/gr.0809 78.108
Taylor, I., Seitz, K., Bennewitz, S., and Walker, J. C. (2013). A simple in vitro method to measure autophosphorylation of protein kinases. Plant Methods 9:22. doi: 10.1186/1746-4811-9-22

Taylor, J. E., and Whitelaw, C. A. (2001). Signals in abscission. New Phytol. 151, 323-339. doi: 10.1046/j.0028-646x.2001.00194.x

Tsuchiya, M., Satoh, S., and Iwai, H. (2015). Distribution of XTH, expansin, and secondary-wall-related CesA in floral and fruit abscission zones during fruit development in tomato (Solanum lycopersicum). Front. Plant Sci. 6:323. doi: 10.3389/fpls.2015.00323

Tucker, M. L., and Yang, R. (2012). IDA-like gene expression in soybean and tomato leaf abscission and requirement for a diffusible stelar abscission signal. AoB Plants 2012:pls035. doi: 10.1093/aobpla/pls035

Tucker, M. L., and Yang, R. (2013). A gene encoding a peptide with similarity to the plant IDA signaling peptide (AtIDA) is expressed most abundantly in the root-knot nematode (Meloidogyne incognita) soon after root infection. Exp. Parasitol. 134, 165-170. doi: 10.1016/j.exppara.2013.03.019

Van de Peer, Y., Fawcett, J. A., Proost, S., Sterck, L., and Vandepoele, K. (2009). The flowering world: a tale of duplications. Trends Plant Sci. 14, 680-688. doi: 10.1016/j.tplants.2009.09.001

van Doorn, W. G. (2001). Categories of petal senescence and abscission: a reevaluation. Ann. Bot. 87, 447-456. doi: 10.1006/anbo.2000.1357

van Doorn, W. G. (2002). Effect of ethylene on flower abscission: a survey. Ann. Bot. 89, 689-693. doi: 10.1093/aob/mcf124

Vanneste, K., Baele, G., Maere, S., and Van de Peer, Y. (2014). Analysis of 41 plant genomes supports a wave of successful genome duplications in association with the Cretaceous-Paleogene boundary. Genome Res. 24, 1334-1347. doi: 10.1101/gr.168997.113

Wang, X., Goshe, M. B., Soderblom, E. J., Phinney, B. S., Kuchar, J. A., Li, J., et al. (2005). Identification and functional analysis of in vivo phosphorylation sites of the Arabidopsis brassinosteroid-insensitive1 receptor kinase. Plant Cell 17, 1685-1703. doi: 10.1105/tpc.105.031393

Woodhouse, M. R., Tang, H., and Freeling, M. (2011). Different gene families in Arabidopsis thaliana transposed in different epochs and at different frequencies throughout the rosids. Plant Cell 23, 4241-4253. doi: 10.1105/tpc.111. 093567

Zanne, A. E., Tank, D. C., Cornwell, W. K., Eastman, J. M., Smith, S. A., FitzJohn, R. G., et al. (2014). Three keys to the radiation of angiosperms into freezing environments. Nature 506, 89-92. doi: 10.1038/nature12872

Zeng, L., Zhang, Q., Sun, R., Kong, H., Zhang, N., and Ma, H. (2014). Resolution of deep angiosperm phylogeny using conserved nuclear genes and estimates of early divergence times. Nat. Commun. 5:4956. doi: 10.1038/ncomms5956

Zhang, N., Zeng, L., Shan, H., and Ma, H. (2012). Highly conserved low-copy nuclear genes as effective markers for phylogenetic analyses in angiosperms. New Phytol. 195, 923-937. doi: 10.1111/j.1469-8137.2012.04212.x

Conflict of Interest Statement: The authors declare that the research was conducted in the absence of any commercial or financial relationships that could be construed as a potential conflict of interest.

Copyright (C) 2015 Stø, Orr, Fooyontphanich, Jin, Knutsen, Fischer, Tranbarger, Nordal and Aalen. This is an open-access article distributed under the terms of the Creative Commons Attribution License (CC BY). The use, distribution or reproduction in other forums is permitted, provided the original author(s) or licensor are credited and that the original publication in this journal is cited, in accordance with accepted academic practice. No use, distribution or reproduction is permitted which does not comply with these terms. 